\title{
Biomarcadores y Marcadores de Imagen de la Enfermedad de Alzheimer
}

\author{
Biomarkers and Image Markers of Alzheimer's Disease
}

Pardi, P.C. ${ }^{\text {; }}$ Santos, G.A.A. ${ }^{3}$; Silva Gois, J.C. ${ }^{3}$ Braz Jr., R.G. ${ }^{4}$ \& Olave, E. ${ }^{5}$

PARDI, P. C.; SANTOS, G. A. A.; SILVA GOIS, J. C.; BRAZ, Jr. R. G. \& OLAVE, E. Biomarcadores y marcadores de imagen de la enfermedad de Alzheimer. Int. J. Morphol., 35(3):864-869, 2017.

RESUMEN: Este artículo presenta un análisis desde el punto de vista bibliográfico de marcadores y biomarcadores de la enfermedad de Alzheimer (EA). Las metodologías usadas fueron los marcadores de imágenes (Resonancia Magnética y Tomografía por emisión de positrones) y biomarcadores de la proteína BA42, de la proteína Tau y de la Apoliproteína E (ALPE). De esta manera, son de importancia los niveles de BA42 disminuidos, la Tau incrementada, los polimorfismos de ALPE y las alteraciones constatadas en los marcadores de imagen, como factores de riesgo esenciales para el desarrollo de la EA. Se realiza una revisión de la literatura con respecto a los hallazgos clínicos de esta enfermedad.

PALABRAS CLAVE: Apolipoproteína; Proteína Tau; Biomarcadores.

\section{INTRODUCCIÓN}

La forma más común de demencia encontrada actualmente es la enfermedad de Alzheimer (EA) que afecta a millones de personas en el mundo (Anoop et al., 2010). La EA es un desorden neurodegenerativo grave del cerebro, de progresión lenta no lineal, siendo caracterizada por la disminución cognitiva progresiva y pérdida de la memoria. En su mayoría, los casos de EA son espontáneos (edad de riesgo > 60 años) y < de 2,5\% son de propensión hereditaria. Aproximadamente 80 millones de personas en el mundo van a sufrir de esta enfermedad en el año 2050 (Blennow et al., 2010). Se sabe que los actuales métodos encontrados en el mercado son ineficaces en el diagnóstico preciso y precoz de la EA y por ello, investigadores han trabajado en nuevas técnicas con el uso de biomarcadores para el diagnóstico satisfactorio de la EA (Santos, 2016).

Continuando con las investigaciones actuales, la medición ELISA de B-amiloide (1-42), Tau total y fosfo-Tau-181 en el líquido cerebroespinal (LCE) es considerado actualmente el método más avanzado, preciso y confiable en el diagnóstico de esta enfermedad (Blennow et al.).

Por lo tanto, el objetivo del presente artículo es la revisión de la literatura respecto de los biomarcadores moleculares y marcadores de imágenes que puedan detectar las manifestaciones de la enfermedad de Alzheimer.

\section{MATERIAL Y MÉTODO}

La revisión de la literatura respecto al tema fue realizada en 2016, basado en las búsquedas en bancos de datos tales como: Science Direct, SciELO, PubMed y Lilacs, utilizando las palabras clave: biomarcadores, marcadores de imágenes, beta-amiloide, fisiopatología de la EA, Apolipoproteína E, proteína Tau, Enfermedad de Alzheimer.

\section{RESULTADOS}

Aspectos generales de la EA. La EA fue presentada por primera vez por el psiquiatra y neuropatologista alemán, Alois Alzheimer en Noviembre de 1906 en el 37 Encuentro de Psiquiatras del Sudeste de Alemania. Alois Alzheimer identificó en la necropsia de una paciente, en el tejido cerebral, la presencia de placas diferentes y enmarañados neurofibrilares (Maurer et al., 1997).

\footnotetext{
${ }^{1}$ Faculdade Anhanguera de Gruarulhos, Kroton Educacional, São Paulo, Brasil.

${ }^{2}$ Centro Universitário Senac, São Paulo, Brasil.

${ }^{3}$ Hospital A.C. Camargo, Brasil.

${ }^{4}$ Biomédico, Brasil.

${ }^{5}$ Facultad de Medicina, Universidad de La Frontera, Chile.
} 
Durante décadas, el estudio realizado por Alzheimer en esa paciente permaneció intacto, sin ningún tipo de intervención y las informaciones casi fueron perdidas dentro del Hospital Clínico de Frankfurt. En 1995, Konrad Maurer, Profesor de Psiquiatría de la Universidad de Frankfurt, encontró la ficha de la paciente, dando inicio a nuevos estudios, más específicos, para comprender mejor la etiología y el diagnóstico de la EA.

En general, la EA de presentación tardía, alrededor de los 60 años, ocurre de forma esporádica, mientras que la EA de presentación precoz, alrededor de los 40 años, muestra relación familiar. Ambas EA, precoz y tardía, son una misma e indindistingible unidad clínica y nosológica (Harman, 1996). Según Chaves (2000) la EA es la causa más frecuente de demencia senil en Brasil. Además, esta enfermedad es el tipo de síndrome demencial que más afecta a los ancianos en el mundo, aproximadamente $60 \%$ de los casos (Kalaria et al., 2008). El diagnóstico en la fase inicial de la enfermedad es importante para retardar el desarrollo de la misma, como ya fue citado, lo que garantizará al paciente y a su entorno un bienestar y calidad de vida (Diamond, 2008).

Actualmente se conoce de la existencia de biomarcadores moleculares que pueden reconocer precozmente, alteraciones asociadas a la EA, entre los cuales fueron investigados en líquido cerebroespinal, sangre y saliva, alteraciones en las concentraciones de proteína Tau total, proteína Tau fosforilada y del péptido Beta Amiloide (Starling, 2012).

Fisiopatología de la EA. Las características fisiopatológicas de la EA se presentan décadas antes de las señales clínicas, mostrando una pérdida progresiva de sinapsis, axones y neuronas, que aumentan antes de la presentación de los primeros síntomas cognitivos, siendo en su mayoría episodios de falta de memoria (Jack et al., 2010). La demencia del tipo Alzheimer se caracteriza por un diagnóstico clínico y patológico combinado, que solo puede ser alcanzado de forma definitiva cuando un paciente satisface los criterios clínicos y presenta en la biopsia cerebral o examen post-mortem las alteraciones histológicas de la enfermedad (innumerables placas neuríticas y enmarañados neurofibrilares en el hipocampo y neocórtex; Chaves).

El factor genético es considerado actualmente como preponderante en la etiopatogenia de la EA entre diversos factores relacionados. Además del componente genético, se han apuntado también como agentes etiológicos a la toxicidad de agentes infecciosos, al aluminio, a los radicales libres de oxígeno, a aminoácidos neurotóxicos y a la presencia de daños en microtúbulos y proteínas asociadas (Mestel, 1996).
Desde el punto de vista neuropatológico, se observa en el cerebro de los individuos con EA una atrofia cortical difusa, la presencia de un gran número de placas seniles y ovillos neurofibrilares, degeneraciones gránulo-vacuolares y pérdida neuronal. Se verifica también un cúmulo de proteína beta-amiloide en las placas seniles y de la microtubulina Tau en los ovillos neurofibrilares. Se cree que la concentración de las placas seniles esté correlacionada al grado de demencia en los afectados por la enfermedad. Trastornos de la transmisión de acetilcolina y acetiltransferasas ocurren con frecuencia en los individuos afectados (Katzman, 1986).

$\mathrm{Al}$ análisis macroscópico, hay una atrofia cortical y central, bilateral y simétrica, donde los surcos y fisuras cerebrales se tornan alargados, generando una pérdida de masa encefálica. Cuando la enfermedad afecta a individuos con menos de 65 años, se observa una mayor atrofia cortical que central, diferente al caso de pacientes con edad superior a ésta última, donde se observa una mayor atrofia central que cortical (Gallucci et al., 2005).

El conocimiento de la fisiopatogenia de la EA es muy importante para el desarrollo de posibles marcadores para el diagnóstico precoz y aparición de nuevas terapias, tratando de determinar el origen de la enfermedad y no apenas los síntomas y sus devastadoras manifestaciones clínicas, como ocurre actualmente (Kalaria et al.).

Diagnóstico Clínico. Según Alzheimer's Association (2014) los síntomas específicos de la EA se basan en: Síntomas clínicos precoces - dificultad en recordar conversaciones recientes, lugares, nombres o eventos, apatía y depresión.

Síntomas tardíos - comunicación deficiente, desorientación, confusión mental, juicio alterado, alteraciones de humor y personalidad, así como dificultad para hablar, engullir y marchar.

A pesar de todos los estudios realizados, el diagnóstico de Alzheimer todavía es obtenido bajo la forma de hipótesis, basándose fuertemente, entre algunos criterios, en evaluaciones del comportamiento. La evaluación cognitiva puede ser iniciada con tests rápidos de rastreo como el mini examen del estado mental y posteriormente complementado con otros tests de memoria, como el test de diseño del reloj (Santos). Sumado a ello, el diagnóstico de demencia se basa en la presencia de déficit de memoria y otras funciones como lenguaje, capacidad de reconocer objetos, organización y capacidad de planificación (Nitrini, 2000).

Diagnóstico por imagen. Desde el punto de vista histopatológico, en la EA ocurre una gran pérdida sináptica y muerte neuronal, principalmente en las regiones cerebra- 
les responsables por las funciones cognitivas, destacándose la corteza cerebral, el hipocampo, el estríado ventral y también la corteza entorrinal. Si se compara la corteza y el hipocampo de un individuo con EA respecto a un individuo normal, en el primero ocurre una importante pérdida neuronal, con una gran reducción de sinápsis, atrofia cortical e hipocampal, además de dilatación de los ventrículos. Con el pasar del tiempo el cerebro disminuye su tamaño en gran proporción, afectándose algunas de sus funciones, entre las cuales se puede mencionar a la corteza, que encoje y daña las regiones involucradas con los pensamientos, planos y recuerdos. Ese encogimiento es principalmente grave en el hipocampo, una región de la corteza que ejerce un papel muy importante en la formación de nuevos recuerdos. Los ventrículos (espacios ocupados por fluido dentro del cerebro) son mayores (Desikan et al., 2009).

Imagen por Resonancia Magnética (IRM). La IRM está basada en diseños probabilísticos de regiones de interés (regions of interest - ROI). La atrofia cerebral y la dilatación ventricular son las mayores alteraciones observadas en la EA (Frisoni et al., 2010). Tales cambios son extensivamente relatados. El principal marcador radiológico de la EA es la atrofia de la región temporal medial, específicamente del volumen de la corteza entorrinal (Moraes, 2010). La progresión de la enfermedad se caracteriza por la afección del giro cingulado posterior y de la corteza temporoparietal de asociación. Las alteraciones de la sustancia blanca son comunes en el individuo anciano y pueden sugerir compromiso de pequeños vasos (Reiman $e t$ al., 2004).

Tomografía por emisión de positrones. La PET (positron emission tomography) produce imágenes tridimensionales o funcionales de procesos del organismo. Su uso en la EA se basa en la evaluación del metabolismo cerebral de la glicosis, vía fluordehoxiglicosis (FDG) o del depósito de la proteína beta-amiloide, via PiB (Pittsburg compound B) (Moraes). En la EA se describe menor captación de glicosis en las regiones temporales mediales y en el cíngulo mientras que en el deterioro cognitivo leve (MCI), la captación está más reducida solamente en la corteza entorrinal. Además, la FDG entrega informaciones útiles sobre la progresión y gravedad de la demencia. Por otro lado, el PiB, que se une a las placas beta-amiloides, sería más útil para el diagnóstico precoz del MCI y para predecir el riesgo de conversión de la enfermedad para EA, basado en la presencia de altos niveles de retención del PiB (Petrie et al., 2009).

Diagnóstico por la apolipoproteína E. En el plasma, la apoE es la principal componente de las lipoproteínas de muy baja densidad (VLDL) y de un grupo de lipoproteínas de alta densidad (HDL), envuelta en la redistribución de triglicéridos y colesterol en diferentes tejidos. El gen apoE humano está localizado en el brazo largo del cromosoma 19 (Mensenkamp et al., 1999).

En los humanos, el cerebro es el segundo sitio de mayor síntesis de apoE, siendo producida principalmente por astrocitos y por la microglia. Otros estudios han sugerido que, por lo menos bajo ciertas condiciones, las neuronas humanas pueden sintetizar apoE en cantidades significativas (Roses et al., 1998).

Comprobando el papel específico de la apoE en la formación de placas amiloides y enmarañados neurofibrilares, existen estudios que muestran que la apoE es uno de los componentes de las placas amiloides cerebrales. La apoE4 promueve la fibrilogénesis in vivo e in vitro del péptido beta-amiloide y la apoE3 se une a la proteína Tau, disminuyendo su tasa inicial de fosforilación y la formación de filamentos (Ojopi et al., 2004).

Variantes de la apoE. En los humanos existen tres alelos principales del gen apoE, resultado de apenas dos alteraciones en el DNA, llamados de e2, e3 y e4. Las isoformas proteícas producidas por esos alelos difieren en la composición de aminoácidos en las posiciones 112 y / o 158 (Weisgraber et al., 1982). El alelo e2 posee el aminoácido cisteína en las dos posiciones de la proteína (aminoácidos 112 y 158), el e3 tiene cisteína en el 112 y una arginina en el 158, siendo este último el alelo más común de la apoE, mientra que el alelo e4 posee arginina en estas dos posiciones. Las otras variantes de la apoE son llamadas de e1, e5 y e7, pero ellas son extremadamente raras.

Considerando la heterogeneidad genética de la EA, con a lo menos cinco o seis genes principales responsables además de otros, probablemente involucrados, se torna difícil realizar una sugerencia genética en base a un único modelo teórico y mendeliano. El gen de la apoE4, considerado un factor de riesgo para la EA, alcanza cerca del $50 \%$ de todos los casos. La subsecuente identificación de los genes directamente responsables por la mayoría de los casos de EA, podrá entonces, permitir la realización de diagnósticos moleculares de predisposición genética a esta afección (Smith, 1999).

Diagnóstico por la Beta-Amiloide (BA). Según ManzanoLeón \& Mas-Oliva (2006) las placas seniles observadas en los exámenes anatomopatológicos de la EA, tienen como su principal componente al péptido Beta-Amiloide. Este es un producto natural del metabolismo de la proteína precursora de amiloide (PPA). Existen varias vías alternativas para la proteolísis de la PPA, entre las cuales la que lleva a la formación de BA. Después de la separación del retículo 
endoplasmático (RE) y del complejo de Golgi, la PPA es entregada al axón, donde a través de transporte axonal rápido es llevada a los terminales sinápticos (Villemagne et al., 2013).

La formación de las placas seniles ocurre cuando una enzima del tipo secretasa, beta-secretasa, fija a la APP y libera un fragmento terminal mayor que el común. Este fragmento es procesado por la gamma-secretasa, ocurriendo la liberación de la beta-amiloide. Su solubilidad es limitada y forma los agregados que son los constituyentes de las fibrillas encontradas en las placas seniles (Cerqueira, 2009).

En el cerebro, el depósito de BA es lento y prolongado, pudiéndose extender por más de dos décadas, lo que puede favorecer intervenciones terapéuticas que puedan modificar el curso de la probable enfermedad neurodegenerativa (Villemagne et al.).

Se han reconocido tres efectos tóxicos sinápticos provocados por la BA: inhibición del potencial de acción en el largo plazo (long-term potentation-LTP), remoción de los receptores sinápticos de glutamato y eliminación de las sinápsis de glutamato. Las sinápsis de glutamato representan entre 85 y $90 \%$ de las sinápsis en la corteza de mamíferos (Cerqueira). Cuando surgen placas de BA en el cerebro, los niveles de BA42 disminuyen en comparación con el líquido cerebroespinal de un individuo saludable, mientras que ocurre aumento de Tau3.

Diagnóstico a través de la proteína TAU. La proteína Tau hace parte de la familia de las proteínas asociadas a los microtúbulo (microtubule-associated proteins-MAP). La principal función de las MAPs es estabilizar los microtúbulos por la agregación de tubulina. El gen que codifica la proteína Tau asociada al microtúbulo posee 16 exones y su transcripción es completa, originando 12 copias de RNAm por splicing alternativo y seis isoformas de proteína. Se trata de una proteína compuesta por 352 a 441 residuos de aminoácidos (Goedert, 2004).

Desde que se descubrió la proteína Tau como componente llave en los enmarañados neurofibrilares (ENF) en la EA, esta proteína pasó a ser objeto de intensas investigaciones. La proteína Tau es causa de otros desordenes neurodegenerativos, además de la EA, conocidas como Tauopatías y estas enfermedades tienen en común grandes cantidades de agregados de proteína Tau (Robert \& Mathuranath, 2007).

En células nerviosas sanas, la proteína Tau se encuentra normalmente en los axones, al contrario de los hallazgos en las tauopatías, en las cuales esta proteína se localiza en el cuerpo celular y en las dentritas. Se puede hallar en forma soluble o insoluble y esta última es identificada en los filamentos helicoidales pareados (FHP), que es el principal componente de los ENF. Los FHPs presentan de 6 a 8 grupos fosfato por molécula de proteína Tau, lo que, en comparación con el grado de fosforilación usual de la proteína Tau en cerebros sanos (en torno de dos grupos fosfato por molécula), permite afirmar que la proteína Tau identificada en los FHPs se encuentra en un estado hiperfosforilado (Brandt et al., 2005).

Hay estudios que señalan que la hiperfosforilación reduce la capacidad de la Tau para estabilizar los microtúbulos, comprometiendo la dinámica microtubular, afectando el transporte intraneuronal, resultando en efectos deletéreos sobre diversos procesos celulares. Cuando la proteína se ve afectada, se altera el transporte axonal, factor vital para la mantención de la homeostasis neuronal. La hiperfosforilación de la proteína Tau favorece la formación de agregados, bloqueando el tráfico intracelular de proteínas neurotróficas y otras proteínas funcionales, resultando en pérdida o disminución en el transporte axonal o dendrítico en las neuronas (Wang \& Liu, 2008).

El aumento de la expresión de la Tau también causa cambios en la morfología celular, retarda el crecimiento y provoca alteraciones importantes en la distribución de organelos transportados por proteínas motoras dependientes de microtúbulos. La hiperfosforilación de la Tau presente en el citosol durante estadios iniciales de degeneración neurofibrilar induce a cambios estructurales que preceden a su agregación. Existen algunas controversias sobre la polimerización de la Tau y su toxicidad (Brandt et al.). Estudios recientes han mostrado que esa agregación de la Tau es tóxica para las células, su polimerización está asociada a la pérdida de la actividad biológica, esencial para promover la estabilidad y la cohesión de los microtúbulos. Sin embargo, la desfosforilación de la Tau hiperfosforilada y de los filamentos helicoidales pareados hacen que la proteína recupere sus actividades biológicas normales (Wang \& Liu).

La proteína Tau también promueve la interacción entre la actina y los neurofilamentos, lo que sugiere una interrelación de los microtúbulos con otros componentes del citoesqueleto. La proteína Tau interactúa con otros organelos citoplasmáticos, permitiendo la unión entre microtúbulos y mitocondrias. Los dominios de proyección $\mathrm{N}$-terminal de la proteína Tau permiten una interacción con la membrana plasmática neuronal (Buée et al., 2000).

Marcadores de la EA. Los biomarcadores son medidas objetivas de un proceso patológico o biológico y pueden ser usados para evaluar el riesgo de la enfermedad o pronósti- 
co, para orientar el diagnóstico clínico o para monitorear las intervenciones terapéuticas. Un biomarcador es objetivamente medido y evolucionado como indicador de un proceso biológico normal, proceso patogénico o respuesta farmacológica a una intervención terapéutica (Blennow et al.). El diagnóstico definitivo de EA sólo puede ser hecho mediante un análisis histopatológico de tejido cerebral postmortem. Las alteraciones histopatológicas incluyen pérdida neuronal en las camadas piramidales de la corteza cerebral y degeneraciones sinápticas intensas, tanto a nivel hipocampal como neocorticales (Braak \& Braak, 1991).

El biomarcador ideal para detectar la EA debe presentar especificidad y sensibilidad, tal como el diagnóstico clínico, además de ser confiable y reproducible, de fácil ejecución, bajo costo y no invasivo, como estudios en sangre, orina, saliva y raspados orales. Exámenes invasivos en piel, biopsias rectales, muestras de médula ósea o líquido cerebroespinal o también biopsia cerebral, se presentan como inconvenientes para la práctica clínica (Bermejo-Pareja et al., 2010).

\section{CONCLUSIONES}

La búsqueda de nuevos biomarcadores depende de estudios para una mayor comprensión de la fisiopatología de la EA. La identificación de biomarcadores puede abrir caminos para nuevos abordajes terapéuticos. Eso es fundamental debido a la incapacidad de modificar de forma eficiente la evolución de la EA, pues actualmente sólo se consigue retardar la velocidad de la progresión de la enfermedad. En esta enfermedad se visualizan marcadores de imágenes como la atrofia cerebral (principalmente en la región temporal), dilatación de los ventrículos y atrofia hipocampal. A través de los biomarcadores, en los pacientes con EA se observa una disminución de la síntesis de alipotroteína E, una mayor cantidad de Beta-amiloide en las placas y un aumento de los enmarañados neurofibrilares agregando las proteínas Tau.

PARDI, P. C.; SANTOS, G. A. A.; SILVA GOIS, J. C.; BRAZ, Jr. R. G. \& OLAVE, E. Biomarkers and image markers of Alzheimer's disease. Int. J. Morphol., 35(3):889-894, 2017.

SUMMARY: This article presents a bibliographical analysis of markers and biomarkers of Alzheimer's disease (AD). The methodologies used were the imaging markers (Magnetic Resonance and Positron Emission Tomography) and biomarkers of the BA42 protein, Tau protein and Apoliprotein E (ALPE). Thus, decreased levels of BA42, increased Tau, ALPE polymorphisms, and alterations in imaging markers are important as risk factors for the development of AD. A review of the literature is made regarding the clinical findings of this disease.

KEY WORDS: Apolipoprotein; Tau Protein; Biomarkers.

\section{REFERENCIAS BIBLIOGRÁFICAS}

Alzheimer's Association. 2014 Alzheimer's disease facts and figures. Alzheimers Dement., 10(2):e47-92, 2014.

Anoop, A.; Singh, P.K.; Jacob, R. S. \& Maji, S. K. CSF Biomarkers for Alzheimer's Disease Diagnosis. Int. J. Alzheimer Dis., pii:606802, 2010.

Bermejo-Pareja, F.; Antequera, D.; Vargas, T.; Molina, J. A. \& Carro, E. Saliva levels of Abeta1-42 as potential biomarker of Alzheimer's disease: a pilot study. B. M. C. Neurol., 10:108, 2010.

Blennow, K.; Hampel, H.; Weiner, M. \& Zetterberg, H. Cerebrospinal fluid and plasma biomarkers in Alzheimer disease. Nat. Rev. Neurol., 6(3):131-44, 2010.

Braak, H. \& Braak, E. Neuropathological stageing of Alzheimer-related changes. Acta Neuropathol., 82(4):239-59, 1991.

Brandt, R.; Hundelt, M. \& Shahani, N. Tau alteration and neuronal degeneration in tauopathies: mechanisms and models. Biochim. Biophys. Acta, 1739(2-3):331-54, 2005.

Buée, L.; Bussière, T.; Bueé-Scherrer, V.; Delacourte, A. \& Hof, P. R. Tau protein isoforms, phosphorylation and role in neurodegenerative disorders. Brain Res. Brain Res. Rev., 33(1):95-130, 2000.

Cerqueira, A. A. B. Estratégias Farmacológicas para as Alterações Precoces do Comportamento da Doença De Alzheimer. Coimbra, Faculdade de Medicina, Universidade de Coimbra, 2009.

Chaves, M. I. F. Diagnóstico Diferencial das Doenças Demenciantes. In: Frolenza, O. V. \& Caramelli, P. (Eds.). Neuropsiquiatria Geriátrica. São Paulo, Atheneu, 2000. pp.81-106.

Desikan, R. S.; Cabral, H. J.; Hess, C. P.; Dillon, W. P.; Glastonbury, C. M.; Weiner, M. W.; Schmansky, N. J.; Greve, D. N.; Salat, D. H.; Buckner, R. L.; Fisch, B. \& Alzheimer's Disease Neuroimaging Initiative. Automated MRI measures identify individuals with mild cognitive impairment and Alzheimer's disease. Brain, 132(Pt. 8):2048-57, 2009.

Diamond, J. Report on Alzheimer's Disease and Current Research. Toronto, Alzheimer Society of Canada, 2008.

Frisoni, G. B.; Fox, N. C.; Jack, C. R Jr.; Scheltens, P. \& Thompson, P. M. The clinical use of structural MRI in Alzheimer disease. Nat. Rev. Neurol., 6(2):67-77, 2010.

Gallucci Neto, J. Tamelini, M. G. G.; \& Forlenza, O. Diagnóstico diferencial das demências. Rev. Psiquiatr. Clin., 23(3):119-30, 2005.

Goedert, M. Tau protein and neurodegeneration. Semin. Cell Dev. Biol., 15(1):45-9, 2004.

Harman, D. A. A hypothesis on the pathogenesis of Alzheimer's disease. Ann. N. Y. Acad. Sci., 786:152-68, 1996.

Jack, C. R. Jr.; Knopman, D. S.; Jagust, W. J.; Shaw, L. M.; Aisen, P. S.; Weiner, M. W.; Petersen, R. C. \& Trojanowski, J. Q. Hypothetical model of dynamic biomarkers of the Alzheimer's pathological cascade. Lancet Neurol., 9(1):119-28, 2010.

Kalaria, R. N.; Maestre, G. E.; Arizaga, R.; Friedland, R. P.; Galasko, D.; Hall, K.; Luchsinger, J. A.; Ogunniyi, A.; Perry, E. K.; Potocnik, F.; Prince, M.; Stewart, R.; Wimo, A.; Zhang, Z. X.; Antuono, P. \& World Federation of Neurology Dementia Research Group. Alzheimer's disease and vascular dementia in developing countries: prevalence, management, and risk factors. Lancet Neurol., 7(9):812-26, 2008.

Katzman, R. Alzheimer's disease. N. Engl. J. Med., 314(15):964-73, 1986. 
Manzano-León, N. \& Mas-Oliva, J. Estrés oxidativo, péptido b-amiloide y enfermedad de Alzheimer. Gac. Méd. Méx., 142(3):229-38, 2006.

Maurer, K.; Volk, S. \& Gerbaldo, H. Auguste D and Alzheimer's disease. Lancet, 349(9064):1546-9, 1997.

Mensenkamp, A. R.; Jong, M. C.; van Goor, H.; van Luyn, M. J.; Bloks, V.; Havinga, R.; Voshol, P. J.; Hofker, M. H.; van Dijk, K. W.; Havekes, L. M. \& Kuipers, F. Apolipoprotein E participates in the regulation of very low density lipoprotein-triglyceride secretion by the liver. J. Biol. Chem., 274(50):35711-8, 1999

Mestel, R. Puttings prions to the test. Science, 273(5272):184-9, 1996.

Moraes, E. N. Incapacidade Cognitiva: Abordagem Diagnóstica e Terapêutica das Demências No Idoso. $2^{\text {nd }}$ ed. Belo Horizonte, Folium, 2010.

Nitrini, R. Epidemiologia da Doença de Alzheimer. In: Frolenza, O. V. \& Caramelli, P. (Eds.). Neuropsiquiatria Geriátrica. São Paulo, Atheneu, 2000. pp.23-34.

Ojopi, E. P. B.; Bertoncini, A. B. \& Dias Neto, E. Apolipoproteína E e a doença de Alzheimer. Rev. Psiquiatr. Clín., 31(1):26-33, 2004.

Petrie, E. C.; Cross, D. J.; Galasko, D.; Schellenberg, G. D.; Raskind, M. A.; Peskind, E. R. \& Minoshima, S. Preclinical evidence of Alzheimer changes: convergent cerebrospinal fluid biomarker and fluorodeoxyglucose positron emission tomography findings. Arch. Neurol., 66(5):632-7, 2009.

Reiman, E. M.; Chen, K.; Alexander, G. E.; Caselli, R. J.; Bandy, D.; Osborne, D.; Saunders, A. M. \& Hardy, J. Functional brain abnormalities in young adults at genetic risk for late-onset alzheimer's dementia. Proc. Natl. Acad. Sci. U. S. A, 101(1):284-9, 2004.

Robert, M. \& Mathuranath, P. S. Tau and Tauopathies. Neurol. India, 55(1):11-6, 2007.

Roses, A. D.; Gilbert, J.; Xu, P. T.; Sullivan, P.; Popko, B.; Burkhart, D. S.; Christian-Rothrock, T.; Saunders, A. M.; Maeda, N. \& Schmechel, D. E. Cis-acting human ApoE tissue expression element is associated with human pattern of intraneuronal ApoE in transgenic mice. Neurobiol. Aging, 19(1 Suppl.):S53-8, 1998.

Santos, G. A. A. A Utilização de Biomarcadores na Doença de Alzheimer. Tese (Doutorado) - Curso de Programa de Doutorado em Biotecnologia e Inovação em Saúde. São Paulo, Universidade Anhanguera de São Paulo, 2016.

Smith, M. A. C. Doença de Alzheimer. Rev. Bras. Psiquiatr., 21(Suppl. 2):3-7, 1999.

Starling, D. S. V. Investigação de Biomarcadores Diagnósticos para a Doença de Alzheimer no Líquido Cefalorraquidiano, na Saliva e na Mucosa Oral. Tese de Doutorado. Minas Gerais, Universidade Federal de Minas Gerais, 2012.

Villemagne, V. L.; Burnham, S.; Bourgeat, P.; Brown, B.; Ellis, K. A.; Salvado, O.; Szoeke, C.; Macaulay, S. L.; Martins, R.; Maruff, P.; Ames, D.; Rowe, C. C. \& Masters, C. L. \& Australian Imaging Biomarkers and Lifestyle (AIBL) Research Group. Amyloid b deposition, neurodegeneration, and cognitive decline in sporadic Alzheimer's disease: a prospective cohort study. Lancet Neurol., 12(4):357-67, 2013.

Wang, J. Z. \& Liu, F. Microtubule-associated protein tau in development, degeneration and protection of neurons. Prog. Neurobiol., 85(2):148$75,2008$.

Weisgraber, K. H.; Innerarity, T. L. \& Mahley, R. W. Abnormal lipoprotein receptor-binding activity of the human $\mathrm{E}$ apoprotein due to cysteinearginine interchange at a single site. J. Biol. Chem., 257(5):2518-21, 1982.

\author{
Dirección para Correspondencia: \\ Dr. Enrique Olave \\ Facultad de Medicina \\ Universidad de La Frontera \\ Temuco \\ CHILE
}

E-mail: enrique.olave@ufrontera.cl

Recibido : 14-01-2017

Aceptado: 19-04-2017 